\title{
PHYSICS OF EXCITONIC QUASIPARTICLES: BEYOND CONVENTIONAL POLARITONS*
}

\author{
Joseph L. BIRMAN, Maurizio ARTONI and Bing-Shen WANG \\ Physics Department, City College of City University of New York, New York 10031, USA
}

\begin{abstract}
:
Some aspects of post-conventional polariton physics are discussed. In the framework of quantum statistical properties we demonstrate that polaritons are intrinsically squeezed. The polariton squeezing is frequency tunable, and can be larger than that in usual atomic systems. The new phonoriton excitation, which gives rise to spectrum reconstruction, is reexamined on the basis of a simple model: threshold behavior, new gap structure and related properties are discussed.
\end{abstract}

\section{Dedication}

We are happy to be able to present this contribution to the Festschrift honoring Professor Vladimir Moiseevich Agranovich on his 60th birthday. It has been the privilege of one of us (JLB) to have known him personally for more than 20 years, and to have followed his work for nearly 30 years. If memory serves, we first met at the famous 1969 Conference on Solid State Theory in Moscow organized by our revered colleague, Professor Ilya Mikhailovich Lifshitz. But I had followed Vladimir's beautiful work some 10 years earlier when, with Professor Vitaly L. Ginzburg [1], he developed the theory of optical properties of solids including spatial dispersion effects. In the years since, I admired his work on excitonic effects on optical properties of semiconductors, on effects due to boundaries and surface waves, and generally on the electrodynamic effects of matter plus radiation. His work has been a model of the energetic pursuit of novel effects in solids and he sets a fine example of professionalism, insofar as he extracts to the last detail the important physical and mathematical consequences of results obtained. To those of my students working on problems of optics I give a standard instruction: read [Volodya] Agranovich's papers! We wish Volodya continued excellent health, and vigor and we look forward to his contributions to our common field of crystal (condensed matter) optics for a very long time to come.

In this paper we would like to sketch some topics of our group's current interest related to extending the polariton concept. Rather than giving a detailed mathematical discussion, which will be presented elsewhere $[2,4]$, we shall indicate the major lines of approach, some results, and some open questions.

\section{Introduction}

The exciton-polariton idea has proven extremely fruitful in the more than 30 years since the introduction of this concept for the coupled electromagnetic (photon) and electronic (exciton) degrees of freedom. (A comprehensive review including historical references is given in ref. [1].) Among the

* This work was supported in part by NASC Contract \#N00019-87-C-0251 and Faculty Research Award of CUNY, PSC \#RF 6-68345. 
aspects of polaritons which have been proved experimentally are those related to the dispersion of the mixed modes, i.e., the determination of frequency-wave vector characteristics and the weak interactions of polaritons with phonons via inelastic scattering experiments. Such experiments as the multiple interference studies, and resonant Brillouin scattering and resonant Raman scattering gave unequivocal confirmation of the existence of polaritons and of their dispersion, and certain of their dynamical properties.

In our recent studies our group's interests have rather turned to some different and for us new and exciting directions within the confines of the study of mixed, propagating modes. We have opened an investigation into the quantum statistical properties of the polaritons, and discovered some quite remarkable features of intrinsic squeezing of polaritons. We are also continuing our studies of progagation of optical pulses in resonant polariton media in order to explore in detail the conditions (parameter space) permitting propagation of pulses under nonlinear optical excitation and in particular possible "polariton-soliton" effects. A new study for us concerns the generalized polariton-type excitations consisting of phonoriton and photoriton, which were (to our knowledge) first introduced by L.V. Keldysh and his collaborators. In the following section we will describe some of our work on these topics.

\section{Polariton squeezing [2]}

Although many aspects of the physics of polaritons have been studied, the statistical properties of the mixed mode have been largely ignored. We have investigated this aspect of the formation of polaritons inside a crystal and we find that polaritons are intrinsically squeezed quasiparticles, and that the amount of squeezing can be varied by frequency tuning, since the squeeze factor depends on the frequency of the polariton. The detection of such squeezing is a topic of our present research effort.

The theory proceeds by considering a slightly generalized Hopfield Hamiltonian for the polariton. Thus we assume that, when an electromagnetic wave propagates in a bounded spatially dispersive nonmagnetic crystal, the propagating polariton excitation consists of the oscillating electromagnetic field clothed by the polarization of the medium; this system can be described by a second-quantized Hamiltonian,

$$
\begin{aligned}
H & =\sum_{k}\left(H_{k}+H_{-k}\right)+\text { h.c. } \\
H_{k} & \equiv E_{k}^{\mathrm{ph}} a_{k}^{+} a_{k}+E_{k}^{\mathrm{exc}} b_{k}^{+} b_{k}+B_{k}^{\prime} a_{k} a_{-k}-C_{k}^{\prime} b_{k} b_{-k}+\mathrm{i} A_{1 k} a_{-k} b_{k}+\mathrm{i} A_{2 k} a_{k}^{+} b_{k}+h_{k}^{0} .
\end{aligned}
$$

Here we have defined $E_{k}^{\mathrm{ph}}=\hbar c k / 2+B_{k}, E_{k}^{\text {exc }}=\hbar \omega_{k} / 2-C_{k}$ and $4 h_{k}^{0}=\left[\hbar c k+\hbar \omega_{k}+2\left(B_{k}-C_{k}\right)\right]$. Excitons, as boson normal modes of the electronic polarization, are represented by the annihilation and creation operators $b_{k}, b_{k}^{+}$; similarly photons are represented by the operators $a_{k}, a_{k}^{+}$. The photon frequency is $c k$ ( $c$ is the velocity of light $/ \sqrt{\varepsilon_{b}}$ ), while that of the exciton is $\omega_{k}=\omega_{0}+\hbar k^{2} / 2 M\left(\omega_{0}\right.$ is the exciton frequency at $k=0 ; M$ is the exciton effective mass). One band of excitons is taken into account; others are included in a background dielectric constant $\varepsilon_{\mathrm{b}}$ independent of frequency and wave vector. We neglect anistropy, damping and loss.

Compared to the standard Hopfield Hamiltonian the above $H_{k}$ includes direct exciton-exciton coupling and coefficients $A_{1 k} \neq A_{2 k}$. 
As is straightforward to show, there is a Bogoliubov transformation which reduces the Hamiltonian (1) to that of a system of uncoupled harmonic oscillator normal modes $\eta_{ \pm k}$ which diagonalize (1). The operators $\eta_{ \pm k}$ are: $\eta_{ \pm k}=x a_{ \pm k}+y b_{ \pm k}^{+}+z a_{\mp k}^{+}+\nu b_{\mp k}$. On the other hand, mode operators

$$
a_{ \pm} \equiv \alpha_{k}\left(\theta_{k}\right) a_{ \pm k}+\mathrm{e}^{2 \mathrm{i} \delta} \beta_{k}\left(\theta_{k}\right) b_{ \pm k}
$$

can be defined such that the exciton-radiation Hamiltonian (1) is put into the form of a standard two-mode squeeze Hamiltonian (see, e.g., ref. [3]): $H_{\mathrm{sq}}\left(\Omega_{k}, \zeta_{k}, \theta_{k}\right)=H_{0}+H_{1}$, where the free part $H_{0}=\Omega_{k}\left(a_{+}^{+} a_{+}+a_{-}^{+} a_{-}\right)$and the quadratic interaction $H_{\mathrm{I}}=2 \zeta_{k}\left(a_{+}^{+} a_{-}^{+}+a_{-} a_{+}\right)$. To accomplish this transformation requires the identifications

$$
\zeta_{k}=B_{k}^{\prime}+C_{k}^{\prime}, \quad \theta_{k}=\sin ^{-1} \sqrt{C_{k}^{\prime} / B_{k}^{\prime}+C_{k}^{\prime}}, \quad \Omega_{k}=2\left(E_{k}^{\mathrm{ph}}+E_{k}^{\mathrm{exc}}\right), \quad \delta=\pi / 4 .
$$

However, using (3), diagonalization based on $\left[H, \eta_{ \pm k}\right]=\varepsilon_{k} \eta_{ \pm k}$ yields a dispersion relation

$$
\varepsilon_{k}^{2}=\Omega_{k}^{2}-4 \zeta_{k}^{2}
$$

The two modes $a_{+}, a_{-}$are themselves certain mixtures of "bare" modes of the photon and exciton polarization field. The eigenenergies $\varepsilon_{k}$ for the modes $\eta_{ \pm k}$ depend upon the exciton and photon energy and the coupling functions $B_{k}, B_{k}^{\prime}, C_{k}, C_{k}^{\prime}$ (eq. 3). These coupling functions can be determined by inverting a measured dispersion curve $\varepsilon_{k}$ for a given material (e.g. CdS, GaAs). When the Hamiltonian is diagonalized and the eigenvector $\{x, y, z, \nu\}$ is found, an identification of the coefficients will give $\{x, y, z, \nu\}$ as $F\left[\Omega_{k}, \rho_{k}, \theta_{k}, \varepsilon_{k}\right]$, i.e., a mapping.

Now let $\left|n_{k}\right\rangle$ be number eigenstates of $H_{0}$, then number eigenstates $\left|n_{k}\right\rangle_{r, \phi}$ of the full Hamiltonian, $H_{0}+H_{\mathrm{I}}$, can be constructed as eigenstates of the number operator $\mu_{ \pm}^{+} \mu_{ \pm}$, where

$$
\mu_{ \pm}=S^{+}(r, \phi) a_{ \pm} S(r, \phi)=a_{ \pm} \cosh r+\mathrm{e}^{2 \mathrm{i} \phi} a_{\mp}^{+} \sinh r
$$

and $S$ is a two-mode squeeze operator [3]. As a consequence, comparing $\mu_{ \pm}$with $\eta_{ \pm k}$ gives $\{x, y, z, \nu\}=G\left[\theta_{k}, r, \phi\right]$. Then, comparing the components $\{x, y, z, \nu\}$ given in $F$ and $G$, we derive an expression for the squeeze factor $r$,

$$
r=\tanh ^{-1}\left(2 \zeta_{k} /\left(\Omega_{k}+\varepsilon_{k}\right)\right)
$$

For $\phi=\pi, r$ is positive. The $k$-dependence of $r$ is understood. Furthermore, since $\left|n_{k}\right\rangle_{r, \phi}=$ $S(r, \phi)\left|n_{k}\right\rangle$, it follows that the energy eigenstates of our polariton system Hamiltonian (1) correspond to a two-mode squeezing of the energy eigenstates $\left|n_{k}\right\rangle$ of $H_{0}$. We then construct particular superpositions of the $\left|n_{k}\right\rangle_{r, \phi}$ and use them to represent Glauber states $\left|\mu_{k}\right\rangle_{r, \phi}$ for a given standing mode of the exciton-photon interacting system (1). These states are squeezed with respect to the Glauber states $\left|\alpha_{k}\right\rangle$ of the free Hamiltonian $H_{0}$. The distinction between $\left|\alpha_{k}\right\rangle$ and $\left|\mu_{k}\right\rangle_{r, \phi}$ plays a significant role in discussing and interpreting the change in frequency from $\Omega_{k}$ to $\varepsilon_{k}\left(H_{0}\right.$ versus $\left.H\right)$ as related to the presence of squeezing [3]. The intrinsic squeezed structure can be displayed by assuming that our model system for $t<0$ is in a state $\left|\alpha_{k}\right\rangle$ and develops into the state $\left|\mu_{k}\right\rangle_{r, \phi}$ for $t>0$, according to the Hamiltonian (1) which describes the time evolution for any $\varepsilon_{k} \neq \Omega_{k}$.

We shall not give details, but simply observe that in terms of time variation, the system is described 
by a wave packet whose shape is time varying. Two quadratures can be introduced, $X_{k}^{p}, X_{k}^{q}$, whose variances $\left|\Delta X_{k}^{p}\right|^{2}$ and $\left|\Delta X_{k}^{q}\right|^{2}$ measure the width of the packet in momentum and coordinate space, respectively. These variances are state dependent. Now if $\left|\alpha_{k}\right\rangle$ is a minimum uncertainty coherent state, and $\left|\mu_{k}\right\rangle_{r, \phi}$ is a squeezed state then in time

$$
\begin{aligned}
& \left\langle\left.\alpha_{k}|| \Delta X_{k}^{q}\right|^{2} \mid \alpha_{k}\right\rangle_{t}=\frac{1}{4}, \\
& { }_{r, \phi}\left\langle\left.\mu_{k}|| \Delta X_{k}^{q}\right|^{2} \mid \mu_{k}\right\rangle_{r, \phi_{t}}=\frac{1}{4} \mathrm{e}^{-2 r}+\frac{1}{2} \sinh 2 r \sin ^{2}\left(\varepsilon_{k} t / \hbar\right),
\end{aligned}
$$

and similarly for $X_{k}^{p}$.

However, for the states $\left|\mu_{k}\right\rangle_{r, \phi}(r \neq 0, \phi=\pi)$ the equality in the Heisenberg Uncertainty Principle*) holds only whenever $\cos \left(2 \varepsilon_{k} t / \hbar\right)= \pm 1$. During its evolution, $\left|\mu_{k}\right\rangle_{r, \phi}$ does not remain a minimum uncertainty state; for a certain time interval its variance, due to squeezing, becomes even smaller than the value set by the minimum uncertainty state, while the conjugate variance becomes larger. Then, over the cycle, the Glauber standing polariton wave excited at the mode $\omega_{k}=\varepsilon_{k} / \hbar$ and $k$ remains the same but its probability distribution changes remarkably.

As a specific example, we study an exciton-photon system in CdS, that is, an A-excitonic polariton in the state $\left|\mu_{k}\right\rangle_{r, \phi}$; we find narrowing of the width of the wavefunction below the constant minimum uncertainty state value, twice during each cycle of the excited mode. To demonstrate this we determine the couplings $B_{k}, B_{k}^{\prime}, C_{k}, C_{k}^{\prime}$. Making the approximation (eq. 3) $\zeta_{k} \cong B_{k}+C_{k}$ and using the parameters $\omega_{0}=20589 \mathrm{~cm}^{-1}, M=0.89 m_{\mathrm{e}}, \varepsilon_{\mathrm{b}}=9.3$, numerical expressions for $B_{k}$ and $C_{k}$ can be found such that the dispersion relation (4) yields the measured energy-wavevector dependence for the upper branch of the A-exciton polariton in CdS. Inverting eq. (5) a numerical expression for $r$ can be derived and this is shown in fig. $1 ; r$ represents the polariton squeeze factor. Using this result we are able to evaluate, e.g. at $t=0$, the ratio of $(6 \mathrm{~b})$ to $(6 \mathrm{a})$, that is, the amount by which the probability density distribution envelope of the polariton field is reduced (squeezed) with respect to the minimum uncertainty state value (see fig. 2). Furthermore, if we denote the time interval during which the squeezing occurs by $\Delta T_{\mathrm{sq}}$, we can also evaluate it as a function of $k$. We find, for instance, that $\Delta T_{\mathrm{sq}}^{\mathrm{CdS}}$ is predicted to be of the order of magnitude of half a femtosecond at the crossing point $k_{0}$ of the bare exciton and bare photon branches.

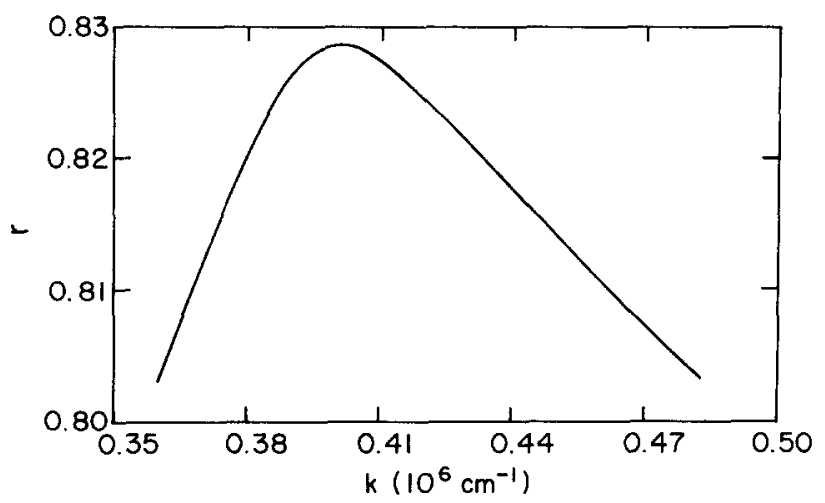

Fig. 1. The wave vector (or frequency) dependence of the squeeze factor $r$ for CdS A-exciton $(\perp \mathrm{c})$ polariton upper-branch states.

${ }^{*)}$ It requires that $\left\langle\left|\Delta X_{k}^{q}\right|^{2}\right\rangle\left\langle\left|\Delta X_{k}^{p}\right|^{2}\right\rangle \geq 1 / 16$. The strict equality holds for minimum uncertainty states. 


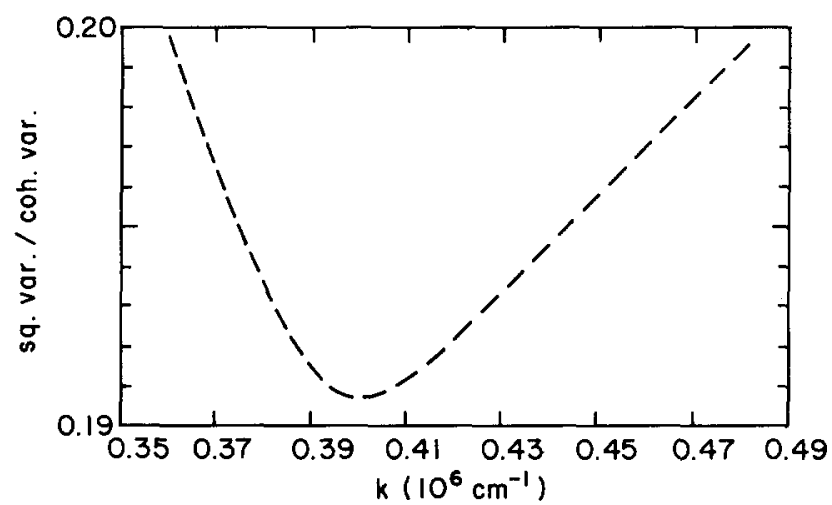

Fig. 2. Squeezing of the probability density distribution for a Glauber polariton standing wave below the minimum uncertainty state value at a given time $(t=0)$ [see eqs $(6)$ ] for the CdS case. Maximum squeezing occurs at the crossing point $k_{0}$ of the bare photon and exciton dispersion curves. For $k$ ranging in the maximum squeezing region the corresponding energy interval is of the order of $0.1 \mathrm{eV}$, on the upper polariton branch dispersion curve].

Examining figs. 1 and 2 convinces one that a quite remarkable amount of intrinsic squeezing (noise reduction) is possible for CdS excitonic polaritons. The experimental investigation of these and other statistical quantum optical properties of the mixed modes will open a new and interesting chapter of polariton physics.

\section{Aspects of phonoriton [4]}

The generalization of the polariton concept to the nonlinear optical regime where the resonant semiconductor is excited by an intense laser (electromagnetic wave) opens another new chapter of generalized polariton physics. (A more extensive discussion is given in ref. [4a, b].)

This generalization of the well-known exciton-polariton was recently introduced by Ivanov and Keldysh to take account of the (non-linear) interaction of intense photon (laser), exciton and phonon systems. This is the "phonoriton". (See ref. [5] and ref. [6] for more recent (1983-87) references.) Starting from a low optical intensity in a spectral region where the exciton-polariton is the natural coupled normal mode of the system, they demonstrated (using a rather dense version of the Schwinger-Keldysh (CTPGF) technique) that a number of very interesting, and qualitatively new phenomena arise when the optical intensity is increased. At high intensity, a new type of quasiparticle exists, and the polariton dispersion equation becomes modified. There is a "reconstruction" which can produce new "gaps" or "pseudogaps" in the spectrum, and correspondingly changes in optical absorption/transmission above the critical intensity.

The high-intensity reconstruction of the polariton spectrum exploited by Keldysh et al. has many antecedents including work of Haken and Schenzle [7] and others.

In the course of our work we observed that phonoriton physics is closely related to the theory of backward resonant Brillouin scattering (RBS) such as the experiment proposed by Brenig, Zeyher and Birman [8].

While the previous theory [8] treats scattering of a polariton excited by an external (laser) field (i.e. initial polaritons) by phonons, the phonoriton formalism treats both such scattering and the coherent rescattering of the scattered polaritons. In a diagrammatic picture, the traditional RBS theory only 

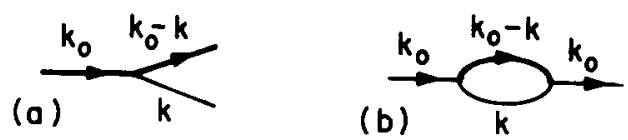

considers the diagram of type (a), while the phonoriton theory considers the diagram of type (b). The usual RBS theory neglects processes (b) because of the short lifetime of phonons. But with increasing intensity of incident photons, the initial polariton mode becomes "macroscopically occupied", and thus the scattering amplitudes of phonon-exciton (polariton) increase accordingly, and processes such as (b) must be taken into account. That is to say, when the strength of this coupling is sufficient to compete with the lifetime and dephasing effects of the phonon, the phonon and scattered polaritons are coherently correlated by the pump polariton, and so spectrum reconstruction occurs.

To illustrate this argument more explicitly, we introduce a Hamiltonian describing the system of interacting photon, exciton and longitudinal phonon,

$$
\begin{aligned}
H= & \sum_{p} \hbar\left[\omega_{p}^{\mathrm{ex}} a_{p}^{+} a_{p}+\omega_{p} b_{p}^{+} b_{p}+\frac{1}{2} \mathrm{i} \Omega_{\mathrm{c}}\left(a_{p}^{+} b_{p}-a_{p} b_{p}^{+}\right)\right] \\
& +\sum_{p} \hbar \Omega_{p} c_{p}^{+} c_{p}+\sum_{p, q}\left[\mathrm{i} M(p-q) b_{p}^{+} b_{q}\left(c_{p-q}+c_{-(p-q)}^{+}\right)+\text {c.c. }\right],
\end{aligned}
$$

where $a_{p}^{+}, b_{p}^{+}, c_{p}^{+}$and $a_{p}, b_{p}, c_{p}$ are creation and annihilation operators for excitons, photons, and phonons, respectively; $\omega_{p}^{e x}$ is the energy of the exciton with momentum $p ; \omega_{p}$ is the energy of the photon; $\Omega_{p}$ that of the phonon; $\Omega_{\mathrm{c}}$ denotes the photon-exciton interaction,

$$
\Omega_{\mathrm{c}}=\omega_{p}^{\mathrm{ex}} \sqrt{4 \pi \beta}
$$

where $\beta$ is the oscillator strength of the exciton; and $\mathrm{i} M(\boldsymbol{p}-\boldsymbol{q})$ is the matrix element of the exciton-phonon interaction. At this moment, we can estimate the values of $\Omega_{\mathrm{c}}$. For CdS, $M$ is of the order of $10^{-4} \mathrm{~cm}^{-1}$ per mode, but $\Omega_{\mathrm{c}}$ is $821 \mathrm{~cm}^{-1}$, so the latter is much stronger.

The first line of (7) can be diagonalized to the form of polaritons (two branches), and the whole Hamiltonian can be written as

$$
H=\sum_{i, p} \omega_{\mathrm{pol}}(\boldsymbol{p}) B_{i, p}^{+} B_{i, p}+\sum_{p} \hbar \Omega_{p} c_{p}^{+} c_{p}+\sum_{i, j, p, q}\left[\mathrm{i} M_{i, j}^{\prime}(p-q) B_{i, p}^{+} B_{j, q}\left(c_{p-q}+c_{-(p-q)}^{+}\right)+\text {c.c. }\right]
$$

where $B_{i, p}^{+}$is the creation operator of the $i$ th branch of polariton with wave vector $p$. The $M_{i, j}^{\prime}$ are renormalized polariton-phonon interaction matrix elements.

Now, suppose a beam of light with frequency $\omega_{0}$ enters the system. Then the wave propagating within the semiconductor is a polariton. For the sake of simplicity we restrict ourselves to the case that there is only one branch of polariton corresponding to the frequency $\omega_{0}$. We denote this wave as $\omega_{0}=\omega_{\text {pol }}\left(k_{0}\right)$ where $\omega_{\text {pol }}(k)$ is the dispersion of the polariton. When the intensity of the beam is high, the polariton mode $k=k_{0}$ will be "macroscopically occupied". But for all other modes, the occupation number is near zero. We divide the system into three parts: incident polariton, phonon and scattered polariton. First we concentrate on the scattered polaritons and phonons. They are coupled by the polariton-phonon interaction $M B_{k_{0}}^{+}$and $M B_{k_{0}}$. If the light is coherent and the intensity high enough, we can with good approximation replace the operator $B_{k_{0}}^{+}$or $B_{k_{0}}$ in the interaction term by their expectation values $\left\langle B_{k_{0}}^{+}\right\rangle$and $\left\langle B_{k_{0}}\right\rangle$, i.e. c-numbers, and also 


$$
\begin{aligned}
& \alpha^{*}(t)=\left(\frac{\varepsilon_{g} V}{2 \pi v_{t} \beta}\right)^{1 / 2} P_{0} \mathrm{e}^{-\mathrm{i} \omega_{k_{0}}{ }^{\prime}}=\alpha_{k_{0}} \mathrm{e}^{-\mathrm{i} \omega_{k_{0}}{ }^{\prime}} \alpha(t)=\left(\frac{\varepsilon_{\mathrm{g}} V}{2 \pi v_{t} \beta}\right)^{1 / 2} P_{0} \mathrm{e}^{-\mathrm{i} \omega_{\boldsymbol{k}_{0}}{ }^{t}}=\alpha_{\boldsymbol{k}_{0}} \mathrm{e}^{-\mathrm{i} \omega_{\boldsymbol{k}_{0}{ }^{\prime}}}, \\
& \alpha_{k_{0}}=\left(\frac{\varepsilon_{\mathrm{g}} V}{2 \pi v_{t} \beta}\right)^{1 / 2},
\end{aligned}
$$

where $v_{t}$ is the group velocity of the polariton, $\beta$ is the oscillator strength of the exciton, $P_{0}$ is the macroscopic polarization amplitude and $\varepsilon_{\mathrm{g}}$ is the dielectric constant of the background. Actually, $\left\langle B_{k_{0}}^{+}\right\rangle$and $\left\langle B_{k_{0}}\right\rangle$ come from the "eigenvalues" of the annihilation operator $B_{k_{0}}$ acting on a coherent state of polariton mode $\boldsymbol{k}_{0}$. After eliminating the time dependent factor $\mathrm{e}^{-\mathrm{i} \omega_{\boldsymbol{k}_{0}}{ }^{t}}$ via a unitary transformation $\exp \left(\mathrm{i} \omega_{k_{0}} B_{k}^{+} B_{k}{ }^{t}\right)$, we can obtain

$$
H_{k}=\sum_{p} \hbar\left(\omega_{p, i}-\omega_{k_{0}}\right) B_{p}^{+} B_{p}+\sum_{p} \hbar \Omega_{p-k} c_{p-k}^{+} c_{p-k}+H_{\mathrm{as}}+H_{\mathrm{s}}
$$

where

$$
H_{\mathrm{as}}=\sum_{p} \mathrm{i} \hbar M^{\prime}(p-k) \alpha_{k_{0}}\left(B_{p}^{+} c_{p-k_{0}}-B_{p} c_{p-k_{0}}^{+}\right), \quad H_{\mathrm{s}}=\sum_{p} \mathrm{i} \hbar M^{\prime}(p-k) \alpha_{k_{0}}\left(B_{p} c_{k_{0}-p}-B_{p}^{+} c_{k_{0}-p}^{+}\right)
$$

are anti-Stokes and Stokes scattering Hamiltonians, respectively. Here

$$
M_{i, j}^{\prime}(p-q)=\sqrt{\psi_{i}^{\mathrm{ex}}(p) \psi_{j}^{\mathrm{ex}}(q)} M(p-q)
$$

is the matrix element between polariton and phonon and $\psi_{i}^{\text {ex }}$ is the weight of the exciton in the polariton in the $i$ th branch. The expression for these factors will be given later.

Now, if we only consider $H_{\text {as }}$, it becomes effectively a simple two-level system. The "scattered polariton" mode $\omega_{p, i}-\omega_{k_{0}}$ (i.e. the energy with respect to incident light) and phonon $\Omega_{p-k_{0}}$ are coupled through the interaction $\sqrt{N_{0}} M_{i, j}^{\prime}\left(p-k_{0}\right)$, and this interaction is proportional to the square root of the strength of the incident macroscopically filled polariton mode. Using a standard procedure, we introduce a linear combination of the annihilation operators of the polariton and phonon,

$$
D=x B_{p}+y c_{p-k}
$$

and demand that

$$
[D, H]=-\hbar \omega D
$$

We can write down the secular equation

$$
\left|\begin{array}{ll}
\omega-\omega_{p}+\omega_{k_{0}} & \mathrm{i} \sqrt{\psi_{i}^{\mathrm{ex}}(p) \psi_{i}^{\mathrm{ex}}(k)} M\left(p-k_{0}\right) \\
-\mathrm{i} \sqrt{\psi_{i}^{\mathrm{ex}}(p) \psi_{i}^{\mathrm{ex}}(k)} M\left(p-k_{0}\right) & \omega-\Omega_{p-k_{0}}
\end{array}\right|=0
$$

The eigenvalue equation is 


$$
\begin{aligned}
& \left(\omega-\omega_{p}+\omega_{k_{0}}\right)\left(\omega-\Omega_{p-k}\right)=\psi_{i}^{\mathrm{ex}}(p) \psi_{i}^{\mathrm{ex}}(\boldsymbol{k}) Q^{2}, \\
& Q=\alpha_{k} M(p-k)=\left(V N_{0}\right)^{1 / 2} M(p-k),
\end{aligned}
$$

where $N_{0}$ is the occupation number of the incident polariton. Note that near the exciton resonance $\psi_{i}^{\text {ex }}(k)$ is practically unity. The solution of this equation gives the reconstructed spectrum, or "phonoriton",

$$
\omega_{1,2}(p)=\frac{1}{2}\left[\omega_{p}+\Omega_{k_{0}-p}-\omega_{k_{0}}\right] \pm \frac{1}{2} \sqrt{\left[\omega_{p}-\Omega_{p-k_{0}}-\omega_{k_{0}}\right]^{2}+\psi^{\mathrm{ex}}(p) Q^{2}} .
$$

A new gap, $\left[\left(\omega_{p}-\Omega_{p-k_{0}}+\omega_{k_{0}}\right)^{2}+\psi^{\text {ex }}(p) Q^{2}\right]^{1 / 2}$, appears, generalizing the gap $\omega_{\ell 0}-\omega_{t 0}$ of the polariton. The new gap depends not only on the properties of the material, but is also proportional to the strength of the pump light. This is a salient feature of the phenomenon.

For the case of Stokes scattering, we can introduce

$$
D=x B_{p}+y c_{k_{0}-p}^{+} .
$$

The basic equation is the same as for the anti-Stokes scattering case but with some changes of the signs,

$$
\left|\begin{array}{ll}
\omega-\omega_{p}+\omega_{k_{0}} & \mathrm{i} \sqrt{\psi_{i}^{\mathrm{ex}}(p) \psi_{i}^{\mathrm{ex}}(\boldsymbol{k})} M\left(\boldsymbol{k}_{0}-p\right) \\
-\mathrm{i} \sqrt{\psi_{i}^{\mathrm{ex}}(p) \psi_{i}^{\mathrm{ex}}(\boldsymbol{k})} M\left(k_{0}-p\right) & \omega+\Omega_{\boldsymbol{k}_{0}-p}
\end{array}\right|=0
$$

The solution is

$$
\omega_{1,2}(p)=\frac{1}{2}\left(\omega_{p}-\Omega_{k_{0}-p}-\omega_{k_{0}}\right) \pm \frac{1}{2} \sqrt{\left(\omega_{p}-\Omega_{\boldsymbol{k}_{0}-p}+\omega_{\boldsymbol{k}_{0}}\right)^{2}-\psi^{\mathrm{ex}}(\boldsymbol{p}) Q^{2}} .
$$

Note that in the case of Stokes scattering there is no real gap.

Actually, neither polariton nor phonon has infinite lifetime, so both $\omega^{\mathrm{ex}}$ and $\Omega$ have nonzero imaginary parts. When we treat these two variables in eqs. (19) and (21) as complex, the solution is also valid. We can combine the resulting expressions for both Stokes and anti-Stokes scattered phonoritons as follows:

$$
\begin{aligned}
& \omega_{1,2}^{ \pm}(p)=\frac{1}{2}\left(\omega_{p} \pm \Omega_{k_{0}-p}-\omega_{k_{0}}+\mathrm{i} \Gamma\right)+(-) \frac{1}{2} \sqrt{\left(\omega_{p} \mp \Omega_{k_{0}-p}+\omega_{k_{0}}+\mathrm{i} \gamma\right)^{2} \pm \psi^{\mathrm{ex}}(p) Q^{2}}, \\
& \Gamma=\gamma_{\mathrm{ph}}+\gamma_{\mathrm{pol}}, \\
& \gamma=\gamma_{\mathrm{ph}}-\gamma_{\mathrm{pol}},
\end{aligned}
$$

where $\gamma_{\mathrm{ph}}$ and $\gamma_{\mathrm{pol}}$ are the inverse lifetimes of phonon and polariton, respectively. The \pm sign in eq. $(22)$ is for Stokes $(+)$ and anti-Stokes $(-)$ scattering, while $+(-)$ is for the two branches in each case. This expression of phonoriton dispersion given by the two-level model is equivalent to the $\tau$ approximation in CTPGF theory [6]. We will discuss some properties of the phonoriton based on this model. 
Let us discuss some conditions for existence of a phonoriton. First, we should have the polaritons propagating inside the substance and they should have enough chance to interact with phonons. So, we need

$$
\operatorname{Re}(k) \gg \operatorname{Im}(k) .
$$

This condition can be satisfied if the frequency $\omega_{0}$ is in the range

$$
\Omega_{\mathrm{c}} \geq \omega_{t}-\omega_{0} \gg\left(\frac{1}{4} \omega_{\mathrm{lt}}^{2}+\gamma \omega_{t}\right)^{1 / 2}-\frac{1}{2} \omega_{\mathrm{lt}},
$$

where $\omega_{1 \mathrm{t}}$ is the difference between longitudinal and transverse bare excitons, and $\gamma$ is the inverse lifetime of the polariton.

In this case the weight factor of the exciton in the polariton $\psi^{\mathrm{ex}}(\boldsymbol{p})$ is given by

$$
\psi^{\mathrm{ex}}(p)=\frac{1}{2}\left(1-\frac{\omega_{p}^{\mathrm{ex}}-\omega_{p}^{\mathrm{ph}}}{\left[\left(\omega_{p}^{\mathrm{ex}}-\omega_{p}^{\mathrm{ph}}\right)^{2}+\Omega_{\mathrm{c}}^{2}\right]^{1 / 2}}\right) \approx 1+4 \frac{\left(\omega_{t}-\omega_{k}\right)^{2}}{\Omega_{\mathrm{c}}^{2}} .
$$

It is practically of the order of unity in the present case.

It is clear from eq. (22) that, when the intensity of the pump light increases from zero, the change of the spectrum also increases. But, if the real part of the splitting is smaller than the imaginary part, the splitting and the spectrum reconstruction cannot manifest themselves. This condition can be expressed as

$$
\Delta \omega>\gamma_{\mathrm{pol}}+\gamma_{\mathrm{ph}} .
$$

$\Delta \omega$ could be obtained from eq. (22). Physically we can understand this as follows: If the lifetime of the phonon and polariton is shorter then the inverse of the scattering and rescattering rate, the scattered polariton and phonon cannot be correlated coherently, so the only physical consequence is the real scattering process, not spectrum anticrossing. In order to determine the pump density at which the phonoriton reconstruction can occur, we turn to the phonon-polariton interaction and give some numerical estimation.

The exciton can interact with longitudinal acoustic phonons through deformation potential interaction. In this case

$$
M(q)=\left(\frac{\hbar}{2 V \rho c_{\mathrm{LA}}}\right)^{1 / 2}|q|^{1 / 2}\left(D_{\mathrm{e}}+D_{\mathrm{h}}\right)
$$

where $V$ is the volume of the crystal, $\rho$ is the density of the material, $c_{\mathrm{LA}}$ is the velocity of the acoustic wave, $D_{\mathrm{e}}$ and $D_{\mathrm{h}}$ are the deformation potential of electron and hole, respectively.

The matrix elements of the Fröhlich interaction between two 1s exciton states can be expressed as

$$
M(q)=\left(\frac{2 \pi \hbar \omega_{0} e^{2}}{V q^{2}}\right)^{1 / 2}\left(\frac{1}{\varepsilon_{\infty}}-\frac{1}{\varepsilon_{0}}\right)^{1 / 2}\left\{\left[1+\left(\frac{1}{2} P_{\mathrm{e}} q a_{\mathrm{B}}\right)^{2}\right]^{-2}-\left[1+\left(\frac{1}{2} P_{\mathrm{h}} q a_{\mathrm{B}}\right)^{2}\right]^{-2}\right\},
$$

where $\omega_{0}$ is the frequency of the LO phonon, $e$ is the charge of the electron, $\varepsilon_{\infty}$ and $\varepsilon_{0}$ are the 
high-frequency and static dielectric constants, respectively, $a_{\mathrm{B}}$ is the Bohr radius of the 1 s exciton. $P_{\mathrm{e}}$ and $P_{\mathrm{h}}$ are two factors defined as follows:

$$
\begin{aligned}
& P_{\mathrm{e}}=m_{\mathrm{e}} /\left(m_{\mathrm{e}}+m_{\mathrm{h}}\right), \\
& P_{\mathrm{h}}=m_{\mathrm{h}} /\left(m_{\mathrm{e}}+m_{\mathrm{h}}\right),
\end{aligned}
$$

where $m_{\mathrm{e}}$ and $m_{\mathrm{h}}$ are the effective masses of electron and hole.

There is another interaction in noncentrosymmetric materials, namely the piezoelectric interaction coupling with TA phonons. For $\mathrm{CdS}$, when the wave vector $q$ is perpendicular to the $Z$-axis, the matrix element is

$$
M(q)=\frac{2 \pi e a_{\mathrm{B}}^{2}}{\varepsilon_{\mathrm{b}}} \mathrm{e}_{15}\left(\frac{h}{2 \rho c_{\mathrm{TA}}}\right)^{1 / 2} q^{3 / 2} \frac{m_{\mathrm{e}}-m_{\mathrm{h}}}{m_{\mathrm{e}}+m_{\mathrm{h}}},
$$

where $e_{15}$ is the piezoelectric constant, $c_{\mathrm{TA}}$ is the velocity of the TA acoustic waves, $\varepsilon_{\mathrm{b}}$ is the dielectric constant of the background.

In all three cases, the interaction increases when the transfer of momentum $|q|$ increases. (For the Fröhlich interaction, the relation is not obvious, but since $|q| \ll a_{\mathrm{B}}$ in the relevant range, we can expand the expression, which then will be proportional to $q^{2}$.) Thus, the greatest splitting will occur in the backscattering geometry.

Let us take $\mathrm{CdS}$ as an example to estimate the value $V M^{2}$ for different scattering mechanisms. Numerical results for this quantity are $10^{-26}, 10^{-28}$, and $10^{-31} \mathrm{~cm}^{-4} \mathrm{eV}^{2}$ for the deformation, Fröhlich and piezoelectric interactions, respectively. In order to detect the appearance of the gap, the splitting should be greater than $10^{-4} \mathrm{eV} ; N_{0}$ should be at least $10^{18-23} \mathrm{~cm}^{-3}$. From $S=N_{0} V_{\mathrm{g}} \hbar \omega$, where $V_{\mathrm{g}}$ is the group velocity of the polariton, we can get $S=10^{18} \cdot 10^{9} \cdot 3 \cdot 10^{-12} \mathrm{erg} / \mathrm{s} \mathrm{cm}^{2}=3 \cdot 10^{8} \mathrm{~W} / \mathrm{cm}$. That means that phonoriton reconstruction is a phenomenon occurring only under very strong radiation. The estimated value of $V_{\mathrm{g}}$ could vary greatly near the crossing or the bottleneck of the polariton. Of course, the value of $S$ can also be small, but in this case the scattering between different branches makes the situation more complex, and we will discuss this elsewhere [46].

From eq. (22), we can also obtain a simple relation,

$$
\operatorname{Im} \omega_{1}+\operatorname{Im} \omega_{2}=\gamma_{\mathrm{ph}}+\gamma_{\mathrm{pol}}
$$

In the case of anti-Stokes scattering, if the splitting (the real part of $\Delta \omega$ ) is much larger than the line width, the lifetime of the phonoriton is of the same order of magnitude as that of the polariton and related phonon.

We can also examine the incident mode and others nearby. They are also coupled via the phonons, and the "coupling constant" is proportional to the density of scattered polariton modes. So these polariton modes are also changed, although the change is not so prominent as for scattered polaritons. If we treat the problem more exactly, the polariton, scattered polariton, and related phonon are all coupled to form the phonoriton. Actually, the scattering, rescattering, and spectrum reconstruction result in nonlinear absorption of the pump light $[4 \mathrm{~b}]$.

It should be noticed that the discussion above uses a rather strong approximation - replacing the operators of the pump mode by c-numbers. This is true only when the polariton is coherent, and of 
course the treatment is similar to the semiclassical one. The limitation of this approximation is that it cannot be used to treat some quantum aspects of the problem, such as coherence and noise and dynamical behavior. On the other hand, this model gives the basic physical picture in the problem and enables us to relate the topic to some existing phenomenological theories of nonlinear phenomena.

\section{Concluding remarks, future directions}

Elsewhere $[4 a, b]$ we discuss in some greater detail the relationship of the phonoriton excitation process to the "inverse Raman scattering". Briefly, inverse Raman scattering is more closely related to an inverse of stimulated Raman scattering. However, the examination in some detail of this relationship is a subject of separate study, which also involves study of the macroscopic, phenomenological limit of the microscopic phonoriton theory given above.

Experimental verification of the existence of phonoritons has been claimed [9] in several semiconductors: $\mathrm{CdS}, \mathrm{HgI}_{2}, \mathrm{PbI}_{2}$. But in our view the situation is not yet settled and we believe additional experiments are required to check or test the theory. Elsewhere we have proposed and analysed two experiments - Nonlinear Resonance Brillouin Scattering and Nonlinear Reflectivity - to measure the reconstructed dispersion due to phonoritons [4b].

Another direction of our study concerns the "quantum optical" properties of phonoritons. A preliminary examination of the Hamiltonian of eq. (7) suggests that the phonoriton quasiparticles are intrinsically squeezed and that the amount of squeezing can be varied by tuning, i.e. using the frequency dependence of the squeeze factor $r$. To measure the intrinsic squeezing of polaritons we proposed and analysed scattering experiments, an adaptation of the homodyne method to the polariton evanescent wave, and a special luminescence study [4a, b].

The brief survey of some active lines should help to support the view that generalized polariton physics is a very active topic with many novel and even unexpected aspects - well worthy of continued research.

\section{References}

[1] V.M. Agranovich and V.L. Ginzburg, Crystal Optics with Spatial Dispersion and Excitons, 2nd Ed. (Springer, Berlin, 1984).

[2] M. Artoni and J.L. Birman, Polariton squeezing, theory and experiment, Quantum Opt. 1 (1989) 91-97; Quantum optical properties of polariton waves, submitted to Phys. Rev.

[3] B.L. Schumaker, Phys. Rep. 135 (1985) 317.

[4] (a) B.-S. Wang and J.L. Birman, Phonoriton: a new elementary excitation in semiconductors under intense pump conditions, in: Festschrift for Prof. Kun Huang (World Scientific, Singapore, 1989/90);

[4] (b) B.S. Wang and and J.L. Birman, Solid State Commun., to be published; Phys. Rev. B, to be published.

[5] A.L. Ivanov and L.V. Keldysh, Sov. Phys. - JETP 57 (1983) 2341.

[6] N.A. Gippius, L.V. Keldysh and S.G. Tikhodeev, Polariton waves near the threshold for stimulated scattering, in: Laser Optics of Condensed Matter, eds J.L. Birman, H.Z. Cummins and A.A. Kaplyanskii (Plenum, New York, 1987) pp. 321-329.

[7] H. Haken and A. Schenzle, Z. Phys. 258 (1973) 231.

[8] W. Brenig, R. Zeyher and J.L. Birman, Phys. Rev. B 6 (1972) 4617.

[9] G.S. Vyskovskii et al., JEPT Lett. 42 (1985) 165;

M.S. Brodin et al., Phys. Stat. Sol. 30 (1983) 735. 